\title{
Use of Sugammadex in a Patient with Amyotrophic Lateral Sclerosis
}

\author{
Ebru Kelsaka ${ }^{a}$ Deniz Karakaya $^{a}$ Eyüp Cağatay Zengin ${ }^{b}$ \\ Departments of ${ }^{a}$ Anesthesiology and ${ }^{b}$ Orthopedics and Traumatology, Faculty of Medicine, Ondokuz Mayıs \\ University, Samsun, Turkey
}

\section{Key Words}

Amyotrophic lateral sclerosis $\cdot$ Rocuronium $\cdot$ Sugammadex

\begin{abstract}
Objective: To report on general anesthesia management in amyotrophic lateral sclerosis. Case Presentation and Intervention: A 47-year-old man presented with fracture of the humerus. The patient was diagnosed with amyotrophic lateral sclerosis. General anesthesia was induced with propofol, rocuronium and remifentanil. After uneventful surgical repair, TOF (train-of-four) ratio reached $>0.90$ at the end of operation. However, muscle strength and tidal volume were inadequate. After sugammadex $2 \mathrm{mg} \mathrm{kg}^{-1}$ i.v. was given, the patient was extubated $120 \mathrm{~s}$ later. Conclusion: This case highlights that rocuronium and sugammadex can be used safely in patients with amyotrophic lateral sclerosis undergoing surgery with general anesthesia.
\end{abstract}

Copyright $\odot 2012$ S. Karger AG, Basel (c) 2012 S. Karger AG, Basel 1011-7571/13/0223-0304\$38.00/0

Karger Open access

This is an Open Access article licensed under the terms of the Creative Commons Attribution-NonCommercial-NoDerivs 3.0 License (www.karger.com/OA-license), applicable to the online version of the article only. Distribution for non-commercial purposes only.

\section{Introduction}

Amyotrophic lateral sclerosis (ALS , Lou Gehrig's disease) is a motor neuron disease characterized by degeneration of upper and lower motor neurons [1]. It is an autosomal-dominant disease common in men between 40 and 50 years of age, with an incidence rate of 2/100,000. Patients often die as a result of respiratory failure 3-5 years after the diagnosis [2].

Sugammadex is an agent used to reverse the effects of steroid-structured nondepolarizing neuromuscular blocking agents such as rocuronium [3]. It acts by rapidly encapsulating steroidal neuromuscular agents to form a stable complex, thereby decreasing the free concentration of the drug from the plasma. The encapsulated complex of sugammadex and neuromuscular blocking agents is freely filtered by the glomerulus into the urine [4]. In recent years, rocuronium-sugammadex has been used frequently, especially in patients with a neuromuscular disease who require the use of nondepolarizing neuromuscular blockers in the implementation of general anesthesia [3].

In this case report, we reviewed general anesthesia management and use of rocuronium-sugammadex in a patient with ALS. 


\section{Case Report}

A 47-year-old, American Society of Anesthesiologists Physical Status (ASA) III, 70-kg man was scheduled for plate-screw fixation using the deltopectoral incision for surgical neck fracture of the humerus at the Department of Orthopedics. The patient's medical history revealed that he had been diagnosed with ALS 2 years prior and had been on baclofen $2 \times 10 \mathrm{mg}$ and riluzole $2 \times 50 \mathrm{mg}$ on a regular basis. Preoperative physical examination showed difficulty in speaking and swallowing. Neurological examination showed anarthria, hypoactive pharyngeal reflexes, grade 4/5 muscle strength in the left lower extremity, and hyperactive deep tendon reflexes. Preoperative 'ALS severity score' was recorded as 26 (speech, 3: limited speech to one word response; swallowing, 7: prolonged time or smaller bite size, lower extremity and walking, 8: difficulty with uneven terrain; upper extremity dressing and hygiene, 8: slow self) [5]. Preoperative complete blood count, biochemistry, chest radiogram and ECG were normal. As a result of consultation with the orthopedic surgeon, we decided to perform general anesthesia to prevent the formation of axillary nerve neuropraxia.

After obtaining informed consent, the patient was taken to the operating room without premedication. Noninvasive blood pressure, $\mathrm{SpO}_{2}$, and ECG monitoring were performed and $0.2 \mu \mathrm{g} \mathrm{kg}^{-1}$ $\mathrm{min}^{-1}$ remifentanil infusion was commenced. Ten minutes after the initiation of remifentanil infusion, propofol $140 \mathrm{mg}(2 \mathrm{mg}$ $\mathrm{kg}^{-1}$ ) was administered for induction of anesthesia. Immediately after the induction, a nasogastric catheter was inserted to prevent aspiration. Neuromuscular transmission was monitored with train-of-four (TOF-Watch SX, Organon, Dublin, Ireland) acceleromyography on the ulnar nerve. After induction of anesthesia, calibration and setup settings of neuromuscular monitor were adjusted before giving rocuronium. The baseline TOF ratio was $>0.90$. A total dose of $20 \mathrm{mg}$ rocuronium was then administered intravenously by titrating in doses of $10 \mathrm{mg}$, and the patient was intubated with a number 8 endotracheal tube when the TOF reached 2 responses. Central body temperature was measured by an esophageal temperature probe.

The patient was put in the semi-sitting position and anesthesia was maintained with sevoflurane (2\%) and remifentanil infusion in a mixture of $50 \%$ oxygen in air. Fifteen minutes after intubation, the TOF ratio increased to 0.75 . Thereafter, an additional $10 \mathrm{mg}$ rocuronium was given for muscle relaxation. After the second dose of rocuronium, the TOF ratio was 0.44 and 0.85 at 30 and $60 \mathrm{~min}$, respectively. During the operation, hemodynamic parameters were within normal limits. Duration of surgery was $75 \mathrm{~min}$. Anesthetic medication was discontinued at the end of the surgery. The TOF ratio was $>0.90$, with $100 \%$ oxygen ventilation before extubation. Although the patient was breathing spontaneously, muscle strength and tidal volume were inadequate and he was unable to open his eyes spontaneously. Sugammadex $2 \mathrm{mg} \mathrm{kg}^{-1}$ i.v. was given. Depth of spontaneous respiration increased and he was able to open his eyes spontaneously after $120 \mathrm{~s}$. Following extubation, the patient was conscious, oriented and cooperative, and was able to move the four extremities with adequate spontaneous breathing. He was transferred to the intensive care unit for postoperative monitoring. The patient's nasogastric catheter was left in place for $24 \mathrm{~h}$. Arterial blood gases, electrolytes, and biochemistry values were nor- mal following extubation and in the intensive care unit. Morphine $4 \times 6 \mathrm{mg}$ was given subcutaneously for $24 \mathrm{~h}$ for postoperative analgesia. The patient was sent to the inpatient service after $24 \mathrm{~h}$.

\section{Discussion}

In our patient with ALS, rocuronium and sugammadex were used safely. Patients with ALS are extremely sensitive to non-depolarizing neuromuscular blockers [6]. Case reports of ALS in the literature are often related to the combined spinal epidural or epidural anesthesia, or general anesthesia management via endotracheal intubation without using nondepolarizing neuromuscular blocking agents [6]. As ALS is not a common disease, there are limited numbers of publications on the use of nondepolarizing neuromuscular blocking agents for general anesthesia management in these patients.

Sugammadex is well designed for patients with neuromuscular system disorders and motor neuron diseases in which careful monitoring of the use of neuromuscular blocking agents is needed [3]. The only report on the use of rocuronium and sugammadex in ALS is that of Wachi et al. [7]. They reported applying only $0.52 \mathrm{mg} \mathrm{kg}^{-1}$ (30 $\mathrm{mg}$ ) rocuronium for anesthesia induction in an 86-yearold patient scheduled for elective colostomy. The TOF response was 3 at the end of the operation. The patient was extubated successfully when $2.1 \mathrm{mg} \mathrm{kg}^{-1}$ sugammadex was applied. There were no postoperative complications.

During anesthesia induction of $0.3 \mathrm{mg} \mathrm{kg}^{-1}(20 \mathrm{mg})$ rocuronium was sufficient to provide the TOF ratio necessary for tracheal intubation. At the end of the operation, TOF ratio was $>0.90$ following the cessation of anesthetics. According to conventional knowledge, TOF ratio should be 0.90 for extubation in patients without motor neuron disease [8]. In our patient, when the TOF ratio was $>0.90$, the depth of respiration was insufficient. Since he was unable to open his eyes spontaneously or respond to verbal commands, we decided to administer sugammadex $2 \mathrm{mg} \mathrm{kg}^{-1}$. After $120 \mathrm{~s}$, the patient's breathing deepened, spontaneous limb movement began, and he opened his eyes. The patient was extubated. Based on this clinical experience, it should be considered that the TOF ratio may be incompatible with the clinical findings in patients with motor neuron diseases such as amyotrophic lateral sclerosis. As there is insufficient information on TOF ratios and the use of rocuronium-sugammadex in motor neuron diseases in the literature, these issues need to be studied further with more case reports. 
Umegaki et al. [9] reported that they applied general anesthesia with sevoflurane and remifentanil without using nondepolarizing neuromuscular blocking agents in patients with ALS who underwent bilateral knee amputation. Endotracheal intubation and extubation were uneventful. Remifentanil is the preferred opioid analgesic because of its short onset time and due to its being well tolerated by these patients.

\section{Conclusion}

This case showed that neuromuscular monitoring must be performed in the management of general anesthesia for patients with ALS. Rocuronium may be chosen for anesthesia induction and maintenance in patient with neuromuscular disease and sugammadex can be used safely in these patients.

\section{References}

$>1$ Turk HM, Ozet A, Kuzhan O, Komurcu F, Arpaci F, Ozturk B, Ataergin S: Paraneoplastic motor neuron disease resembling amyotrophic lateral sclerosis in a patient with renal cell carcinoma. Med Princ Pract 2009; 18 : 73-75.

72 Chou SM, Norris FH: Amyotrophic lateral sclerosis: lower motor neuron disease spreading to upper motor neurons. Muscle Nerve 1993;16:864-869.
3 Unterbuchner C, Fink H, Blobner M: The use of sugammadex in a patient with myasthenia gravis. Anaesthesia 2010;65:302-305.

4 Hunter JM, Flockton EA: The doughnut and the hole: a new pharmacological concept for anaesthesists. Br J Anaesth 2006;97:123-126.

5 Hillel AD, Miller RM, Yorkston K, McDonald E, Norris FH, Konikow N: Amyotrophic lateral sclerosis severity scale. Neuroepidemiology 1989;8:142-150.

-6 Iwanaga S, Kitamura T, Ogawa M, Yamada Y: Anesthetic management of laparotomy for a patient with amyotrophic lateral sclerosis: a case report. Masui 2008;57:987-989.
7 Wachi M, Uehara K, Fujinaka W, Takatori $\mathrm{M}$, Tada K: The use of sugammadex in a patient with amyotrophic lateral sclerosis. Masui 2011;60:1408-1410.

8 Claudius C, Viby-Mogensen J: Acceleromyography for use in scientific and clinical practice. Anesthesiology 2008;108:11171140.

$>9$ Umegaki H, Tagami N: Anesthetic management of an ALS patient with remifentanil. Masui 2008;57:1139-1142. 